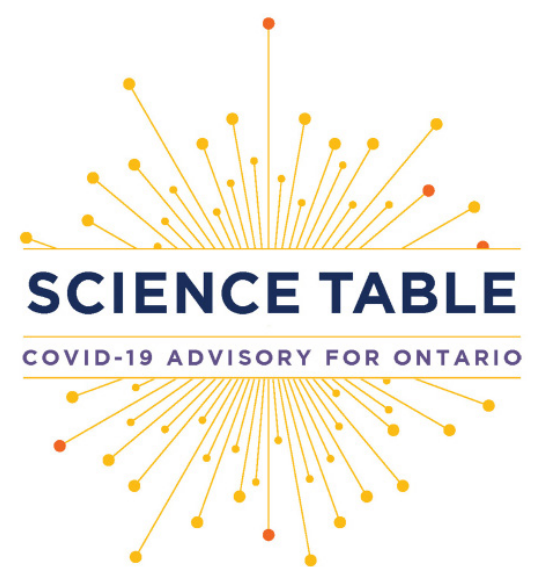

Version: $\mathbf{1 . 0}$

Published: July 16, 2021

Citation: Rochon PA, Brown KA, Huynh T, et al. Ontario's community-dwelling older adults who remain unvaccinated against COVID-19. Science Briefs of the Ontario COVID-19 Science Advisory Table. 2021;2(37). https:// doi.org/10.47326/ocsat.2021.02.37.1.0

Author Affiliations: The affiliations of the members of the Ontario COVID-19 Science Advisory Table can be found at https:// covid19-sciencetable.ca/.

Declarations of Interest: The declarations of interest of the members of the Ontario COVID-19 Science Advisory Table, its Working Groups, or its partners can be found at https:// covid19-sciencetable.ca/. The declarations of interest of external authors can be found under additional resources at https://doi. org/10.47326/ocsat.2021.02.37.1.0

About Us: The Ontario COVID-19 Science Advisory Table is a group of scientific experts and health system leaders who evaluate and report on emerging evidence relevant to the COVID-19 pandemic, to inform Ontario's response. Our mandate is to provide weekly summaries of relevant scientific evidence for the COVID-19 Health Coordination Table of the Province of Ontario, integrating information from existing scientific tables, Ontario's universities and agencies, and the best global evidence. The Science Table summarizes its findings for the Health Coordination Table and the public in Science Briefs.

The Congregate Care Setting Working Group is a group of internationally recognized researchers with expertise in older people living in congregate care settings. The Working Group evaluates emerging scientific evidence related to congregate care settings to inform Ontario's response to the COVID-19 pandemic. The Working Group reports its findings to the public and the Science Table. Its findings are also summarized in Science Briefs.

Correspondence to: Secretariat of the Ontario COVID-19 Science Advisory Table (info@covid19-sciencetable.ca)

Copyright: 2021 Ontario COVID-19 Science Advisory Table. This is an open access

\section{Ontario's Community-Dwelling Older Adults Who Remain Unvaccinated Against COVID-19}

Paula A. Rochon, Kevin A. Brown, Tai Huynh, Rachel Savage, Jennie Johnstone, Pamela Leece, Sudeep S. Gill, Pat Armstrong, Peter Tanuseputro, David M. Kaplan, Rachel Strauss, Wei Wu, Hannah Chung, Peter Jüni, Andrew P. Costa, Nathan M. Stall on behalf of the Ontario COVID-19 Science Advisory Table and the Congregate Care Setting Working Group

\section{Key Message}

COVID-19 vaccination rates among community-dwelling Ontarians aged 65 years and older are lowest in neighbourhoods at highest risk of SARS-CoV- 2 infection, those that have the highest material deprivation, and those that are most ethnically diverse.

Lower rates of vaccination were most pronounced among older adults who had no regular contact with a primary care physician.

Between April 26, 2021 and June 7, 2021, Ontarians aged 80 years and older had the lowest increase in first dose vaccination among all eligible older adults in Ontario, suggesting that vaccination rates in this age group may have plateaued.

Community-dwelling older adults remain at disproportionately high risk of hospitalization and death due to COVID-19, and efforts should be made to maximize vaccination in this population.

\section{Summary}

\section{Background}

As Ontario continues its COVID-19 vaccination program, it remains critical that older adults receive vaccines as they are at highest risk of COVID-19 hospitalization and death. Nearly all (97\%) older Ontarians living in long-term care (LTC) homes are now fully vaccinated. Yet, 93\% of Ontario's older adult population live in the community. Those aged 80 years and older, who are at very high risk of poor COVID-19 outcomes, continue to have a lower vaccination rate than those living in LTC homes, and not all of them have an assigned primary care physician to help guide their care and access to COVID-19 vaccination.

\section{Questions}

Who are the Ontario community-dwelling older adults who have not received their first dose of a COVID-19 vaccine as of June 7, 2021, and what improvement has there been in first dose vaccination rates between April 26 and June 7, 2021?

Is having a primary care physician associated with COVID-19 vaccination in communitydwelling older adults?

What are the characteristics of neighbourhoods where older adults who remain unvaccinated against COVID-19 live?

\section{Findings}

In Ontario, there were 2,696,565 older adults (65 years and older) living in the community, as of January 1,2021 , and the majority (53.8\%) were women. The overall 
document distributed under the terms of the Creative Commons Attribution License, which permits unrestricted use, distribution, and reproduction in any medium, provided that the original work is properly cited.

The views and findings expressed in this Science Brief are those of the authors and do not necessarily reflect the views of all of the members of the Ontario COVID-19 Science Advisory Table, its Working Groups, and its partners. first dose vaccination rate for these older adults living in the community was $83 \%$ as of June 7, 2021. Between April 26 and June 7, 2021, first dose vaccination rates increased among all eligible Ontarians, yet the rate of increase was lowest in those aged 80 years and older. Older adults living in neighbourhoods with the highest risk of SARS-CoV-2, with the highest material deprivation, and the most ethnic diversity had the lowest vaccination rates. The vaccination rate in older adults in Ontario who were rostered to a primary care practice was $40 \%$ higher than in those without a primary care physician.

\section{Interpretation}

There are a substantial number of community-dwelling older Ontarians who have yet to receive their first dose of COVID-19 vaccine, and first dose vaccination rates appear to be plateauing. This is concerning since age is one of the strongest risk factors for COVID-19 hospitalization and death.

Innovative and tailored solutions are needed to increase the vaccination rate among this vulnerable population to prevent morbidity and mortality. Efforts are underway to provide curated lists of their vaccinated patients to family physicians in patientenrolled models; however, this will not address the lower vaccination rate among those with no regular primary care physician. Potential solutions include scaling up efforts with primary care physicians, pharmacies, mobile vaccination clinics for homebound individuals and those residing in naturally occurring retirement communities (NORCs), as well as door-to-door outreach to engage with unvaccinated older adults in culturallyand language-appropriate ways, focusing on high-risk neighbourhoods.

\section{Background}

Older age is one of the greatest risk factors for COVID-19 mortality. ${ }^{1}$ An estimated 93\% of the 6.8-million Canadians aged 65 years and older live in their homes in the community, with the remainder living in congregate care settings such as long-term care (LTC) and retirement homes. Canadian national data from June 9, 2021 shows that 16,719 ( $65.4 \%$ ) of all fatal COVID-19 cases were among adults 80 years of age and older and most of them (55.9\%) were women $(n=9,347) .^{2}$

Eight weeks after the start of Ontario's COVID-19 vaccination rollout, 92\% of LTC home residents were vaccinated with at least one vaccine dose leading to an estimated relative reduction in SARS-CoV- 2 incidence of $89 \%$ and COVID-19 mortality of $96 \% .{ }^{3}$ We know less about the success of Ontario's COVID-19 vaccine rollout among communitydwelling older adults living in the community. ${ }^{3}$ There have also been concerns about the inaccessibility of the vaccine rollout for some older adults. ${ }^{4}$ Appointments at mass immunization clinics require online booking or long-wait times by telephone, and popup clinics operate on a drop-in basis which can result in lengthy and often queues that are designed for specific age groups. There may also be language and transportation barriers. These challenges may be insurmountable for older adults who lack adequate social support networks, including access to a regular primary care physician to assist them with this process. These challenges may be even greater for those living alone or not speaking English.

\section{Questions}

Who are the Ontario community-dwelling older adults who have not received their first dose of a COVID-19 vaccine as of June 7, 2021, and what improvement has there been in first dose vaccination rates between April 26 and June 7, 2021?

Is having a primary care physician associated with COVID-19 vaccination in communitydwelling older adults?

What are the characteristics of neighbourhoods where older adults who remain 
unvaccinated against COVID-19 live?

\section{Findings}

In Ontario, there were 2,696,565 older adults (65 years and older) living in the community as of January 1,2021 . The majority of these individuals $(53.8 \%)$ were women, and $24.5 \%$ were 80 years of age or older. The overall first dose COVID-19 vaccination rate for this group was $83 \%$ as of June 7,2021 .

\section{Vaccination Coverage Among Those 80 Years of Age and Older}

Among all Ontario age groups, vaccination rates have improved least between April 26 and June 7, 2021 for those 80 years of age and older. In the six-week period between April 26 and June 7, 2021, vaccination rates increased among all eligible Ontarians, yet this increase was lowest in those aged 80 years and older. Those aged 80 years and above are most at risk and continue to have a lower vaccination rate than those in LTC homes $(97 \%)$. Further, this oldest group had the lowest increase in vaccination rate among all older age groups over the six-week period.

\begin{tabular}{lccccccccc}
\hline & \multicolumn{3}{c}{ June 7, 2021 } & \multicolumn{3}{c}{ April 26, 2021 } & \multicolumn{3}{c}{ Change in vaccination rate } \\
\hline & Men & Women & Overall & Men & Women & Overall & Men & Women & Overall \\
Overall & $82.6 \%$ & $83.4 \%$ & $83.0 \%$ & $71.4 \%$ & $73.4 \%$ & $72.5 \%$ & $11.2 \%$ & $10.0 \%$ & $10.5 \%$ \\
Age (years) & & & & & & & & & \\
$65-69$ & $80.8 \%$ & $82.4 \%$ & $81.7 \%$ & $60.5 \%$ & $63.9 \%$ & $62.2 \%$ & $20.3 \%$ & $18.5 \%$ & $19.5 \%$ \\
$70-74$ & $83.4 \%$ & $84.1 \%$ & $83.8 \%$ & $73.8 \%$ & $75.4 \%$ & $74.7 \%$ & $9.6 \%$ & $8.7 \%$ & $9.1 \%$ \\
$75-79$ & $84.4 \%$ & $84.8 \%$ & $84.6 \%$ & $78.4 \%$ & $78.9 \%$ & $78.6 \%$ & $6.0 \%$ & $5.9 \%$ & $6.0 \%$ \\
$80+$ & $82.6 \%$ & $83.0 \%$ & $82.8 \%$ & $78.7 \%$ & $78.7 \%$ & $78.7 \%$ & $3.9 \%$ & $4.3 \%$ & $4.1 \%$ \\
\hline
\end{tabular}

Table 1. Rates of COVID-19 Vaccination Among Ontario Community-Dwelling Older Adults, by Sex and Age as of June 7, 2021, Compared to April 26, 2021

Table 1 presents data describing COVID-19 vaccination rate among older adults in Ontario by sex (male and female) and age groups. This table compares vaccination rates as of June 7, 2021, to those as of April 26, 2021. Data were obtained from linked health administrative datasets using unique encoded identifiers and analyzed at ICES. We excluded those living in LTC homes. Information on the administration of COVID-19 vaccines was obtained from provincial COVaxON data through April 26, 2021 and again on June 7, 2021.

\section{Community-Dwelling Older Adults Without an Assigned Primary Care Physician}

Community-dwelling older adults without an assigned primary care physician had lower covid-19 vaccination rates. Table 2 describes the assignment of Ontario community-dwelling older adults to primary care physicians by sex and age group. The majority $(82.7 \%)$ of older adults were rostered to a primary care enrollment model (i.e., Family Health Group or Family Health Organization), a percentage that was slightly higher in women (women $=83.4 \%$ vs. men $=81.9 \%$ ). An additional $11.5 \%$ of older adults had a solo primary care practitioner. A total of 156,096 (5.8\%) of Ontario community-dwelling older adults had no contact with a primary care physician in the prior two years and were classified as having no regular primary care physician.

\begin{tabular}{lccc}
\hline & $\begin{array}{c}\text { No regular primary care physician } \\
(\mathbf{n = 1 5 6 , 0 9 6 )}\end{array}$ & $\begin{array}{c}\text { Assigned primary care physician } \\
(\mathbf{n = 3 0 9 , 5 2 7 )}\end{array}$ & $\begin{array}{c}\text { Rostered to a primary care practice } \\
(\mathbf{n}=\mathbf{2 , 2 3 0 , 9 4 2 )}\end{array}$ \\
\hline $\begin{array}{l}\text { Overall } \\
\text { Sex }\end{array}$ & $5.8 \%$ & $11.5 \%$ & $82.7 \%$ \\
$\quad$ Women & $5.3 \%$ & & $83.4 \%$ \\
Men & $6.4 \%$ & $11.3 \%$ & $81.9 \%$ \\
Age (years) & & $11.7 \%$ & $81.4 \%$ \\
$65-69$ & $6.7 \%$ & & $83.2 \%$ \\
$70-74$ & $5.7 \%$ & $11.9 \%$ & $84.2 \%$ \\
$75-79$ & $5.0 \%$ & $11.1 \%$ & $82.8 \%$ \\
$80+$ & $5.3 \%$ & $10.8 \%$ & $11.9 \%$ \\
\hline
\end{tabular}

Table 2. Primary Care Physician Assignment Among Ontario Community-Dwelling Older Adults

Table 2 presents the assignment of Ontario community-dwelling older adults to a primary care physician categorized by type of primary care physician and stratified by sex (male and female) and age (years). Assignment to a primary care physician was categorized into three groups. Individuals rostered to a primary care practice through a specific primary care enrollment model (e.g., Family Health Group or Family Health Organization) were categorized as being "rostered to a primary care practice." Older adults not identified as being rostered but who received care from a primary care physician within two-years prior to June 7, 2021 were categorized as having an "assigned primary care physician." Those without a visit to a primary care physician within two-years were categorized as having "no regular primary care physician." 
Figure 1 illustrates COVID-19 vaccination rates among community-dwelling older adults by primary care physician assignment, sex, and age as of June 7,2021 . The change in first dose vaccination rate by primary care physician assignment between April 26, 2021 and June 7, 2021 is listed in Appendix 1. Only 45.9\% of older adults with no regular primary care physician had received one dose of COVID-19 vaccine, compared to $76.8 \%$ of older adults with an assigned primary care physician and $86.5 \%$ for those rostered to a primary care practice. Similar trends were observed for both men and women, and for all age groups. The negative impact of not having an assigned primary care physician or being rostered to a primary care practice on vaccination rates was greatest among those who were 80 years of age and older.

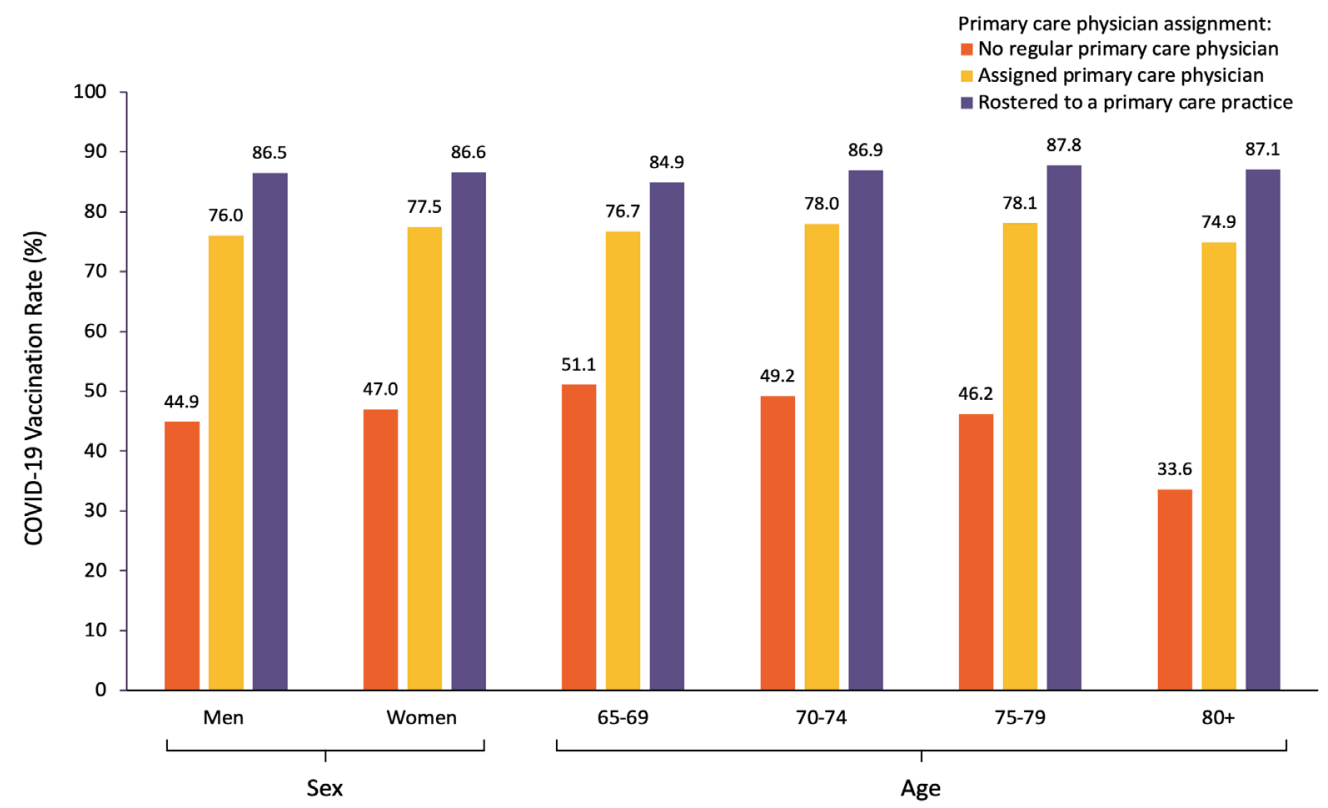

Figure 1. COVID-19 Vaccination Rates by Primary Care Assignment Among Ontario Community Dwelling Older Adults as of June 7, 2021, by Sex and Older Age Group

Figure 1 presents COVID-19 vaccination rates among Ontario community-dwelling older adults within the three categories of primary care physician assignment as well as by sex (male, female) and age group (65-69, 70-74, 75-79, $80+$ years) as of June 7, 2021.

COVID-19 vaccination rates were lower for those living in neighbourhoods with the highest risk of SARS-CoV-2 infection, which also have the most material deprivation and highest ethnic diversity. ${ }^{5-7}$

Table 3 compares the first dose COVID-19 vaccination rates of older adults by deciles of neighbourhood risk of SARS-CoV-2 infection. Overall, vaccination rates were lower in older adults residing in neighbourhoods with a higher risk of SARS-CoV-2 infection. This trend was most pronounced among individuals with no regular primary care physician. In this group, vaccination rates varied from a low of $28.4 \%$ in neighbourhoods with the highest risk of SARS-CoV-2 infections to a high of $62.5 \%$ in neighbourhoods with the lowest risk of SARS-CoV-2 infections. 
Decile 1 = high incidence of SARS-CoV-2 infection and Decile 10 = low incidence of SARS-CoV-2 infection

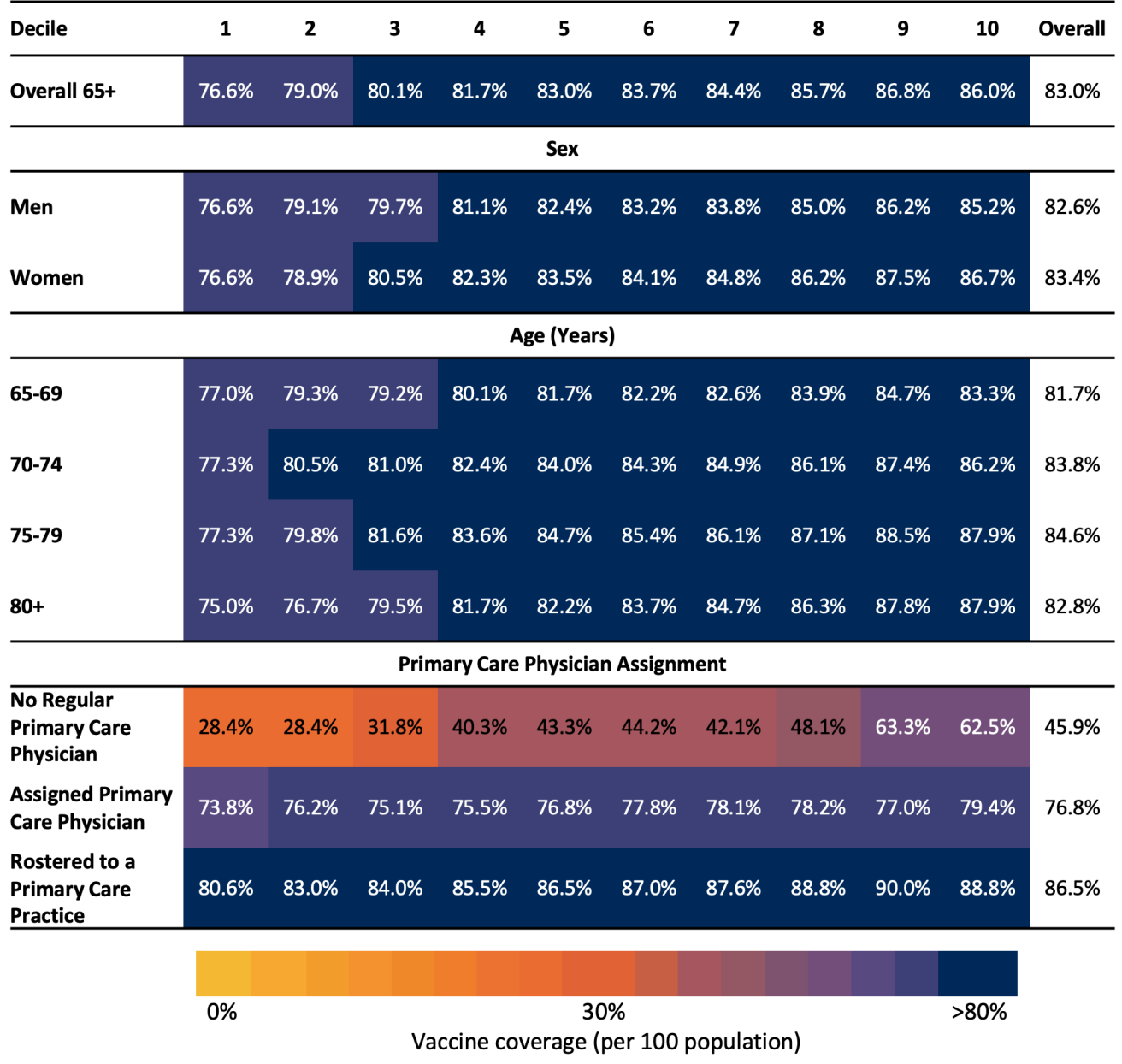

Table 3. Rates of COVID-19 Vaccination Among Ontario Community-Dwelling Older Adults by Neighbourhood-Level Risk of SARS-CoV-2 on June 7, 2021

Table 3 presents the neighbourhood risk of SARS-CoV-2 infection by sex, age group, and primary care physician assignment. For all individuals included, the last recorded postal code in the registered persons data base (RPDB) was used to determine their neighbourhood (defined by the forward sortation area (FSA), the first 3 characters of the postal code). The number of individuals residing in each FSA was used as the denominator for vaccination rates. Eight FSAs with less than 200 individuals were excluded from the analysis. We used a combination of datasets (Ontario Laboratory Information System (OLIS), Public Health Case and Contact Management (CCM) Solutions, and testing data from laboratories within the COVID-19 Diagnostic Network) to determine the cumulative incidence of SARS-CoV-2 infections between January 23, 2020 and March 28, 2021 for each FSA. We ranked FSAs based on their cumulative incidence of SARS-CoV-2 infection among residents living outside of LTC facilities within each FSA and grouped FSAs into deciles based on this rank order.5,6 Each decile has 10\% of the Ontario population ( 1.5 million individuals) and represents the neighbourhood risk of SARS-CoV-2, where $1=$ highest incidence neighbourhoods and $10=$ lowest incidence neighbourhoods. Decile 1 includes neighbourhoods with the highest cumulative incidence of SARS-CoV-2 infection, whereas Decile 10 includes neighbourhoods with the lowest cumulative incidence of SARS-CoV-2 infection. The primary care assignment (no regular primary care, assigned primary care physician, or rostered to a primary care practice) was also described by neighbourhood SARS-CoV-2 infection risk. The colours range from yellow for the lowest rates of COVID-19 vaccination to blue for the highest rates of COVID-19 vaccination. ${ }^{5}$

Table 4 explores first dose vaccination rates within quintiles of neighbourhood-level material deprivation. Material deprivation is a dimension of the Ontario Marginalization Index. ${ }^{7}$ Vaccination rates were lowest in neighbourhoods with higher material deprivation; this pattern was consistent for men and women, for all age groups, and for older adults with a primary care physician. Vaccination rates for older adults with no primary care physician assigned remained low, regardless of neighbourhood-level material deprivation. 


\begin{tabular}{|c|c|c|c|c|c|c|c|c|c|c|}
\hline \multicolumn{11}{|c|}{ Quintile 5 = highest material deprivation and Quintile 1 = lowest material deprivation } \\
\hline & \multicolumn{5}{|c|}{ June 7, 2021} & \multicolumn{5}{|c|}{ April 26, 2021} \\
\hline Quintile & 5 & 4 & 3 & 2 & 1 & 5 & 4 & 3 & 2 & 1 \\
\hline Overall 65+ & $78.8 \%$ & $82.3 \%$ & $83.7 \%$ & $84.6 \%$ & $85.5 \%$ & $64.8 \%$ & $70.3 \%$ & $72.8 \%$ & $75.1 \%$ & $78.1 \%$ \\
\hline \multicolumn{11}{|c|}{ Sex } \\
\hline Men & $77.9 \%$ & $81.8 \%$ & $83.2 \%$ & $84.2 \%$ & $85.2 \%$ & $62.9 \%$ & $69.0 \%$ & $71.8 \%$ & $74.2 \%$ & $77.3 \%$ \\
\hline Women & $79.5 \%$ & $82.7 \%$ & $84.1 \%$ & $84.9 \%$ & $85.8 \%$ & $66.3 \%$ & $71.4 \%$ & $73.8 \%$ & $76.0 \%$ & $78.7 \%$ \\
\hline \multicolumn{11}{|c|}{ Age (Years) } \\
\hline 65-69 & $76.4 \%$ & $80.6 \%$ & $82.4 \%$ & $83.7 \%$ & $84.7 \%$ & $52.1 \%$ & $58.5 \%$ & $62.3 \%$ & $65.8 \%$ & $70.4 \%$ \\
\hline 70-74 & $79.2 \%$ & $83.1 \%$ & $84.4 \%$ & $85.6 \%$ & $86.1 \%$ & $66.1 \%$ & $72.5 \%$ & $75.1 \%$ & $77.7 \%$ & $80.2 \%$ \\
\hline 75-79 & $80.9 \%$ & $84.2 \%$ & $85.3 \%$ & $85.8 \%$ & $86.6 \%$ & $72.3 \%$ & $77.0 \%$ & $79.3 \%$ & $80.8 \%$ & $82.7 \%$ \\
\hline $80+$ & $79.7 \%$ & $82.2 \%$ & $83.4 \%$ & $83.7 \%$ & $85.0 \%$ & $74.1 \%$ & $77.5 \%$ & $79.1 \%$ & $80.4 \%$ & $82.0 \%$ \\
\hline \multicolumn{11}{|c|}{ Primary Care Physician Assignment } \\
\hline $\begin{array}{l}\text { No Regular } \\
\text { Primary Care } \\
\text { Physician }\end{array}$ & $47.3 \%$ & $48.1 \%$ & $47.5 \%$ & $44.1 \%$ & $42.3 \%$ & $34.9 \%$ & $36.8 \%$ & $36.8 \%$ & $34.9 \%$ & $35.0 \%$ \\
\hline $\begin{array}{l}\text { Assigned Primary } \\
\text { Care Physician }\end{array}$ & $72.8 \%$ & $76.3 \%$ & $77.4 \%$ & $78.8 \%$ & $80.1 \%$ & $58.8 \%$ & $64.7 \%$ & $67.5 \%$ & $69.7 \%$ & $72.9 \%$ \\
\hline $\begin{array}{l}\text { Rostered to a } \\
\text { Primary Care } \\
\text { Practice }\end{array}$ & $82.7 \%$ & $85.7 \%$ & $86.9 \%$ & $87.7 \%$ & $88.7 \%$ & $68.4 \%$ & $73.4 \%$ & $75.8 \%$ & $78.1 \%$ & $81.1 \%$ \\
\hline & $0 \%$ & & Vaccin & overa & $\begin{array}{l}0 \% \\
\text { (per } 10\end{array}$ & pu & & & $\%$ & \\
\hline
\end{tabular}

Table 4. Rates of COVID-19 Vaccination Among Ontario Community-Dwelling Older Adults within quintiles of Neighbourhood-Level Material Deprivation, by Age, Sex and Levels of Healthcare Access as of April 26, 2021 and June 7, 2021

Table 4 presents the neighbourhood-level material deprivation by sex, age group, and primary care physician assignment as of April 26, 2021 and June 7, 2021. Material deprivation is a dimension of the Ontario Marginalization Index and is derived from census variables at the dissemination area level. ${ }^{7}$ Each quintile represents different neighbourhood-level material deprivation, where 1 = lowest material deprivation and $5=$ highest material deprivation. Material deprivation is closely connected to poverty and it refers to the inability of individuals and communities to access and attain basic material needs. The indicators included in this dimension measure income, quality of housing, educational attainment and family structure characteristics. ${ }^{7}$

Table 5 explores first dose rates of vaccination across the quintiles of neighbourhood level ethnic diversity. Among those residing in the neighbourhoods with the most diversity, vaccination rates were lower compared with those residing in neighbourhoods with the least diversity. This same pattern was observed irrespective of sex, age, and primary care physician assignment, although, it was most pronounced among older adults with no regular primary care physician. In this group, vaccination rates were $24.7 \%$ in the most diverse neighbourhoods increasing to $64.4 \%$ in the least diverse neighbourhoods. 


\begin{tabular}{|c|c|c|c|c|c|c|c|c|c|c|}
\hline \multicolumn{11}{|c|}{ Quintile 5 = most ethnic diversity and Quintile 1 = least ethnic diversity } \\
\hline & \multicolumn{5}{|c|}{ June 7, 2021} & \multicolumn{5}{|c|}{ April 26, 2021} \\
\hline Quintile & 5 & 4 & 3 & 2 & 1 & 5 & 4 & 3 & 2 & 1 \\
\hline Overall 65+ & $76.0 \%$ & $81.8 \%$ & $85.3 \%$ & $86.7 \%$ & $86.7 \%$ & $66.0 \%$ & $73.5 \%$ & $76.2 \%$ & $75.4 \%$ & $73.1 \%$ \\
\hline \multicolumn{11}{|c|}{ Sex } \\
\hline Men & $75.9 \%$ & $81.3 \%$ & $84.8 \%$ & $86.1 \%$ & $85.9 \%$ & $65.7 \%$ & $72.7 \%$ & $75.2 \%$ & $74.0 \%$ & $71.2 \%$ \\
\hline Women & $76.1 \%$ & $82.2 \%$ & $85.7 \%$ & $87.3 \%$ & $87.4 \%$ & $66.2 \%$ & $74.2 \%$ & $77.0 \%$ & $76.7 \%$ & $74.7 \%$ \\
\hline \multicolumn{11}{|c|}{ Age (Years) } \\
\hline 65-69 & $76.7 \%$ & $80.7 \%$ & $83.7 \%$ & $84.6 \%$ & $84.2 \%$ & $62.0 \%$ & $66.7 \%$ & $66.5 \%$ & $61.5 \%$ & $56.2 \%$ \\
\hline 70-74 & $77.3 \%$ & $82.7 \%$ & $85.8 \%$ & $87.1 \%$ & $87.1 \%$ & $67.8 \%$ & $75.4 \%$ & $78.3 \%$ & $78.0 \%$ & $75.6 \%$ \\
\hline 75-79 & $76.7 \%$ & $83.0 \%$ & $87.1 \%$ & $88.6 \%$ & $88.6 \%$ & $69.1 \%$ & $77.6 \%$ & $82.2 \%$ & $83.2 \%$ & $82.3 \%$ \\
\hline $80+$ & $73.1 \%$ & $81.2 \%$ & $85.4 \%$ & $87.7 \%$ & $88.2 \%$ & $67.2 \%$ & $77.1 \%$ & $81.7 \%$ & $84.4 \%$ & $84.8 \%$ \\
\hline \multicolumn{11}{|c|}{ Primary Care Physician Assignment } \\
\hline $\begin{array}{l}\text { No Regular } \\
\text { Primary Care } \\
\text { Physician }\end{array}$ & $24.7 \%$ & $36.9 \%$ & $47.9 \%$ & $57.4 \%$ & $64.4 \%$ & $18.9 \%$ & $29.4 \%$ & $38.4 \%$ & $44.7 \%$ & $49.2 \%$ \\
\hline $\begin{array}{l}\text { Assigned Primary } \\
\text { Care Physician }\end{array}$ & $74.1 \%$ & $76.6 \%$ & $77.9 \%$ & $79.0 \%$ & $79.2 \%$ & $63.2 \%$ & $67.9 \%$ & $69.2 \%$ & $68.1 \%$ & $66.6 \%$ \\
\hline $\begin{array}{l}\text { Rostered to a } \\
\text { Primary Care } \\
\text { Practice }\end{array}$ & $80.4 \%$ & $85.5 \%$ & $88.4 \%$ & $89.4 \%$ & $89.4 \%$ & $70.1 \%$ & $77.1 \%$ & 79.1\% & $78.0 \%$ & $75.6 \%$ \\
\hline
\end{tabular}

Table 5. Rates of COVID-19 Vaccination Among Ontario Community-Dwelling Older Adults Within Quintiles of Neighbourhood-Level Ethnic Diversity, by Age, Sex, and Primary Care Physician Assignment as of April 26, 2021 and June 7, 2021

Table 5 presents the neighbourhood-level ethnic diversity by sex, age group, and primary care physician assignment as of April 26, 2021 and June 7, 2021. Ethnic diversity is a dimension of the Ontario Marginalization Index and is derived from census variables at the dissemination area level. ${ }^{7}$ Ethnic diversity refers to high area-level concentrations of people who are recent immigrants and/or people belonging to a 'visible minority' group. ${ }^{7}$ Each quintile represents different neighbourhood-level ethnic diversity, where 1 = least ethnic diversity and $5=$ most ethnic diversity.

Figure 2 shows the unvaccinated rate by primary care physician assignment and the proportion unvaccinated with their first dose in Ontario communitydwelling older adults by primary care physician assignment as of June 7, 2021. 


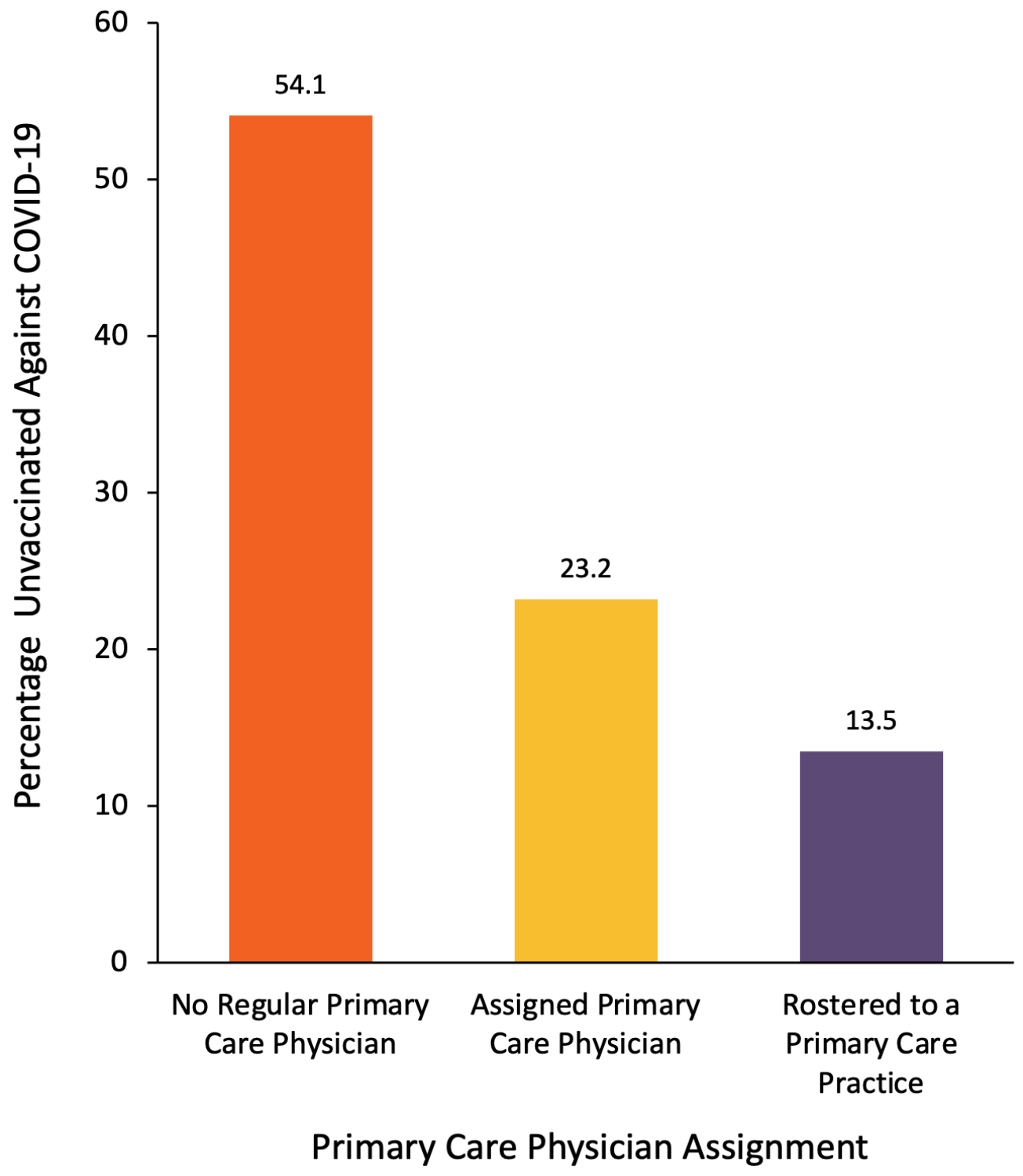

Figure 2a. Proportion Unvaccinated Among Ontario Community-Dwelling Older Adults by Primary Care Physician Assignment

Figure 2 represents the unvaccinated rate among Ontario primary care physician assignment and the proportion of unvaccinated Ontario community-dwelling older adults by primary care physician assignment as of June 7, 2021.

An estimated $54.1 \%$ of older adults without a regular primary care physician are unvaccinated compared to only $13.5 \%$ of older adults rostered to a primary care practice. Yet, because most older adults in the province are rostered to a primary care practice, the largest percentage of unvaccinated older adults $(65.8 \%)$ are still cared for within a primary care practice. 


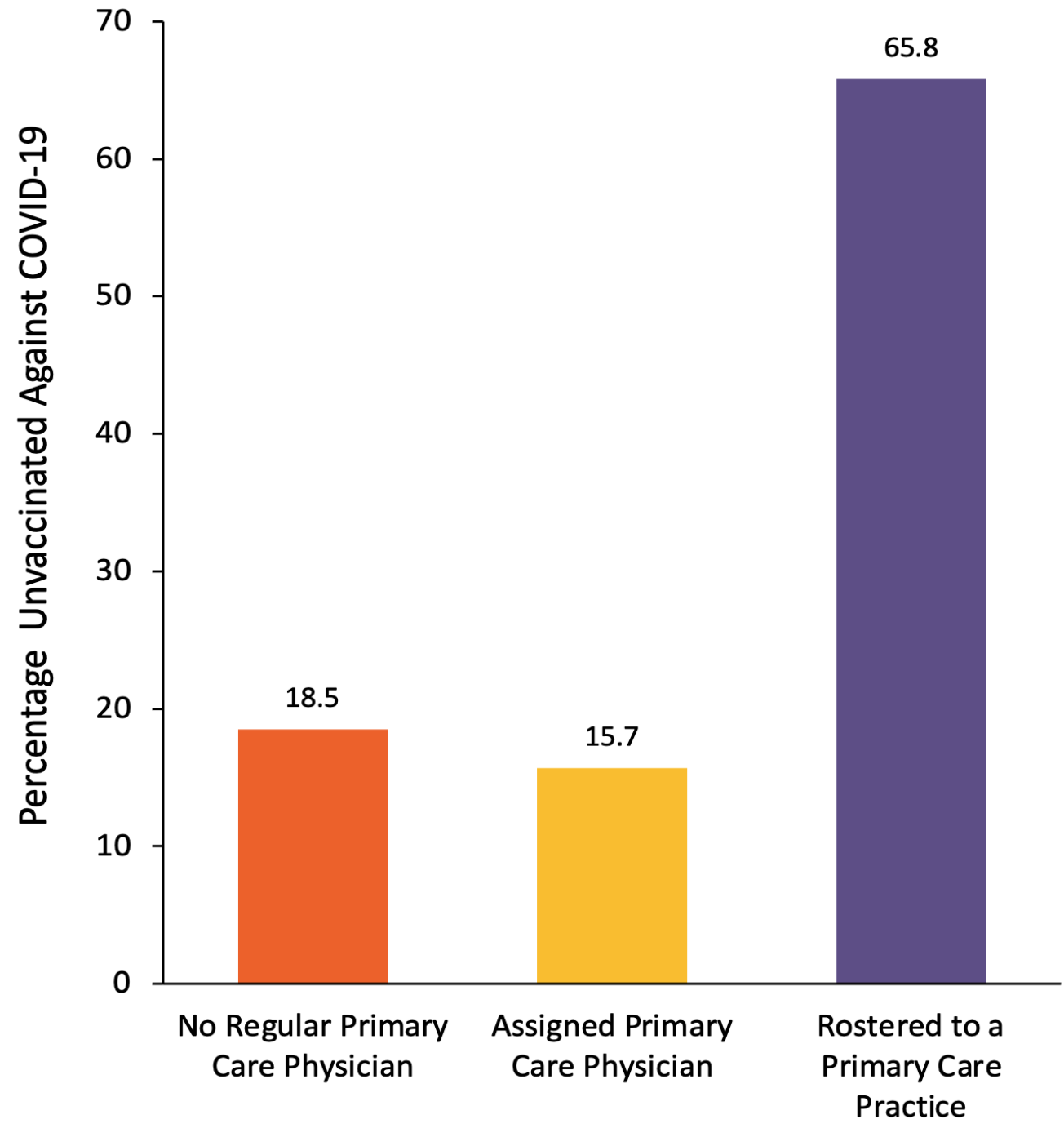

\section{Primary Care Physician Assignment}

Figure 2b. Proportion Unvaccinated Among Unvaccinated Ontario Community-Dwelling Older Adults by Primary Care Physician Assignment as of June 7, 2021

Figure 2 represents the unvaccinated rate among Ontario primary care physician assignment and the proportion of unvaccinated Ontario community-dwelling older adults by primary care physician assignment as of June 7, 2021.

\section{Interpretation}

While the number of unvaccinated community-dwelling older adults decreased over the six-week period between April 26 and June 7, 2021, it is concerning that vaccination rates among those aged 80 years and above appear to be plateauing, especially since they are at very high risk for poor outcomes following COVID-19 infection. Specifically, there has only been a $4 \%$ improvement in first dose vaccination rates among this age group during the study time period. Further, the majority of this unvaccinated older age group are women.

To date, first dose COVID-19 vaccination rates among Ontarians aged 65 years and older were lowest in neighbourhoods at highest risk of SARS-CoV-2 infection, those that have the most material deprivation, and neighbourhoods that were most ethnically diverse. This indicates residual inequities in COVID-19 vaccination, despite the Ontario hotspot vaccination strategy.

These inequities were most pronounced among the $6 \%$ of older adults who had no regular primary care physician. Older adults without a primary care physician may miss 
the benefit of having their primary care physician assist them through the vaccination process, for both first and second doses.

Access may be a major reason why older adults, particularly women in the oldest age group, remain unvaccinated. Most older people are not vaccine hesitant; ${ }^{8}$ they simply may be unaware of how to access the vaccine, unable to manage the booking process, or unable to get to a vaccination clinic to be immunized. Access may be a particular issue for older women who are more likely than men to live alone and may not have someone to care for them, making it more difficult to organize a vaccine appointment and get to the vaccination site. We do know that among LTC homes in Ontario, 97\% of residents are vaccinated. ${ }^{9}$ These findings suggest that vaccination rates for older adults in the community may be much higher if the vaccine was made more accessible to them.

While it is important to look at the inequity in access to vaccination experienced by those without a primary care physician, it is also important to recognize that the vast majority of older adults living in the community are part of a primary health practice. As such, among all unvaccinated older adults, the largest unvaccinated group (65.8\%) are cared for within a rostered primary care practice. Therefore, tactics to target vaccinations to the unvaccinated older adults require increased collaboration with primary care providers who have important roles in addressing vaccine hesitancy in their primary care practices. This means moving from providing vaccinations at mass vaccination sites to working with primary care physicians to identify those in their practices who are unvaccinated and provide these older adults with vaccine access through smaller mobile outreach clinics, administration in physician's offices, and when needed in individual homes. ${ }^{10}$

For primary care physicians and practices to support their older patients in getting vaccinated, they need accurate and up-to-date lists of their unvaccinated patients. At present, this essential information is not available to primary care physicians and asking physicians to call all their patients to determine who is and is not vaccinated is not feasible. Primary care physicians will need support to do this tailored vaccine outreach and to make vaccines available to their older unvaccinated patients. Family physicians caring for patients rostered to a primary care practice will receive curated lists of their patients who have been vaccinated. The fact that the Pfizer vaccine can now be stored in fridges for up to 31 days as well as the large number of Moderna vaccines now available, ${ }^{11,12}$ combined with improved access and accurate, up-to-date lists of unvaccinated patients will facilitate making vaccines more available from the offices of primary care practitioners and for outreach to older adults in their practice who may require home visits.

For the $18.5 \%$ of unvaccinated older adults without a primary care physician, other tactics are required. First, mobile outreach clinics have been very successful in bringing vaccines to the large numbers of older people living in naturally occurring retirement settings (NORCs) who may not be able to get to mass vaccination clinics. ${ }^{13}$ These efforts need to continue. NORCs are apartment, condo, co-op, and social housing buildings with at least $30 \%$ of their residents being 65 years of age and above, and with at least 50 older persons per building. They differ from LTC and retirement homes which are purpose-built for older adults. ${ }^{14}$ To ensure that NORC mobile outreach clinics are targeting buildings where older adults remain unvaccinated, NORC-level vaccine coverage data have been prepared and shared with Ontario's 34 Public Health Units (PHUs).

Further, some unvaccinated community-dwelling older adults may be unable to leave their homes or reluctant to leave their homes due to concerns about becoming infected with SARS-CoV-2. This illustrates the importance of working together with community agencies and knocking on doors to bring vaccines to individuals with 
information provided in the languages that they speak, especially since we found that older adults residing in Ontario neighbourhoods with the highest ethnic concentration had the lowest first dose vaccination rates. ${ }^{15}$

Finally, there is a population of older adults who do not have a regular primary care provider and who may be socially isolated. This includes many older women, who may be without someone to advocate on their behalf to help arrange for vaccinations.

COVID-19 has made existing gaps in our healthcare system visible, particularly for older Ontarians. Our findings demonstrate that a substantial proportion of communitydwelling older adults, particularly women in the oldest age groups, remain unvaccinated with even a first dose against COVID-19. These results also show that a group of these most vulnerable older adults have no primary care physician, and this group is much more likely to be unvaccinated. Our findings can inform the development of urgently needed tactics to improve COVID-19 vaccination among Ontario community-dwelling older adults who remain at a high risk of severe COVID-19 outcomes.

\section{Methods Used for This Science Brief}

\section{Study Population and Design}

We used a cross-sectional study design to quantify the COVID-19 vaccination rate among all community dwelling older women and men in Ontario.

\section{Data Sources}

Data for this analysis were obtained through linked health administrative datasets using unique encoded identifiers and analyzed at ICES. ICES is an independent, non-profit research institute whose legal status under Ontario's health information privacy law allows it to collect and analyze health care and demographic data, without consent, for health system evaluation and improvement. We used the Registered Person's Database (RPDB) to identify individuals alive and eligible for the Ontario Health Insurance Plan (OHIP) and aged 65 or older as of January 1, 2021. We then excluded individuals without a health care encounter (date of last contact) within the last 9 years and those identified as living in a LTC home. We identified these individuals using recent health service transactions (e.g., OHIP physician billings, Ontario Drug Benefit (ODB) Plan claims) and Resident Assessment Instrument (RAI) assessments from the Continuing Care Reporting System (CCRS). For all individuals included, the last recorded postal code in the RPDB was used to determine their FSA (for definition of incidence deciles, or their dissemination area, for the ON-MARG index). Eight FSAs with less than 200 individuals were excluded from the analysis.

We used a combination of datasets (Ontario Laboratory Information System (OLIS), Public Health Case and Contact Management (CCM) Solutions and testing data from laboratories within the COVID-19 Diagnostic Network) to determine the cumulative incidence of SARS-CoV-2 cases between January 23, 2020 (the date of the first reported case within these data) and March 28, 2021 for each FSA. We ranked FSAs based on their cumulative incidence of SARS-CoV-2 infection among residents living outside of LTC homes within each FSA and grouped FSAs into deciles based on this rank order.5, 6 Each decile has $10 \%$ of the Ontario population ( 1.5 million individuals) and represents the neighbourhood risk of SARS-CoV-2, where $1=$ highest incidence neighbourhoods and $10=$ lowest incidence neighbourhoods.

Information on the administration of COVID-19 vaccines was obtained from provincial COVaxON data and was reported as of both April 26, 2021 and June 7, 2021. Physician billing claims under Ontario's Health Insurance Plan (OHIP) along with data from the Client Agency Program Enrolment (CAPE) dataset were ascertained to assess contact with a primary care provider. 


\section{Outcomes}

The primary outcome was the proportion of older adults receiving at least one dose of a COVID-19 vaccine.

\section{Variables}

COVID-19 vaccination rates were reported by various individual-level factors including age, sex, and primary care assignment. Assignment to a primary care physician was categorized into three groups. Individuals rostered to a primary care practice through a specific primary care enrollment model (e.g., Family Health Group or Family Health Organization) were categorized as being "rostered to a primary care practice." Older adults not identified as being rostered but who received care from a primary care physician within two-years (prior to June 7, 2021 for latest results and April 26, 2021 for earlier results) were categorized as having an "assigned primary care physician." Those without a visit to a primary care physician within two-years were categorized as having "no regular primary care physician."

We ranked FSAs based on their cumulative incidence of COVID-19 infection among residents living outside of LTC facilities within each FSA and grouped FSAs into deciles based on this rank order. ${ }^{5,6}$ Each decile has $10 \%$ of the Ontario population ( 1.5 million individuals) and represents the neighbourhood risk of SARS-CoV-2, where $1=$ highest incidence neighbourhoods and $10=$ lowest incidence neighbourhoods. Material deprivation and ethnic concentration (diversity) are two dimensions of the Ontario Marginalization Index and are derived from census variables at the dissemination area level. ${ }^{7}$ Material deprivation is closely connected to poverty, and it refers to inability for individuals and communities to access and attain basic material needs. The indicators included in this dimension measure income, quality of housing, educational attainment and family structure characteristics. ${ }^{7}$ Ethnic concentration refers to high area-level concentrations of people who are recent immigrants and/or people belonging to a 'visible minority' group.'

\section{Analysis}

We reported vaccination rates by the variables detailed above. Subgroup analyses were performed by age groups, sex, and levels of assignment to a primary care physician.

\section{Author Contributions}

PAR, KAB, TH, and NMS conceived the Science Brief. PAR wrote the first draft. HC conducted the analyses. All authors revised the Science Brief critically for important intellectual content and approved the final version.

This work is supported by the Applied Health Research Question (AHRQ) program at ICES, which is funded by the Ontario Ministry of Health. For more information on AHRQ and how to submit a request, please visit www.ices.on.ca/DAS/AHRQ. The work also is supported by the Ontario Health Data Platform (OHDP), a Province of Ontario initiative to support Ontario's ongoing response to COVID-19 and its related impacts. Parts of this material are based on data and information compiled and provided by the Ontario Ministry of Health, the Canadian Institute for Health Information, and Public Health Ontario (PHO). We would like to acknowledge PHO for access to case level data from their Case and Contact Management (CCM) system and COVID-19 laboratory data, as well as assistance with data interpretation. We also thank the staff of Ontario's PHUs who are responsible for COVID-19 case and contact management and data collection within CCM. The analyses, conclusions, opinions, and statements expressed herein are solely those of the authors and do not reflect those of ICES, OHDP, $\mathrm{PHO}$ or the funding or data sources; no endorsement is intended or should be inferred. 


\section{References}

1. Williamson EJ, Walker AJ, Bhaskaran K, et al. Factors associated with COVID-19related death using OpenSAFELY. Nature 2020; 584(7821): 430-6.

2. Government of Canada. COVID-19 daily epidemiology update. https://healthinfobase.canada.ca/covid-19/epidemiological-summary-covid-19-cases. html?stat=num\&measure=total_last7\&map=pt\#a2.

3. Brown KA, Stall NM, Vanniyasingam T, et al. Early impact of Ontario's COVID-19 vaccine rollout on long-term care home residents and health care workers. Science Briefs of the Ontario COVID-19 Science Advisory Table. 2021;2(13). https://doi. org/10.47326/ocsat.2021.02.13.1.0.

4. Stall NM, Nakamachi Y, Chang M, et al. Mobile in-home COVID-19 vaccination of Ontario homebound older adults by neighbourhood risk. Science Briefs of the Ontario COVID-19 Science Advisory Table. 2021;1(19). https://doi.org/10.47326/ ocsat.2021.02.19.1.0.

5. Update on COVID-19 Projections. Ontario COVID-19 Science Advisory Table and Modelling Consensus Table. May 20, 2021. https://covid19-sciencetable.ca/ wp-content/uploads/2021/05/Update-on-COVID-19-Projections_2021.05.20_ English-2.pdf.

6. Mishra S, Stall NM, Ma H, et al. A vaccination strategy for Ontario COVID-19 hotspots and essential workers. Science Briefs of the Ontario COVID-19 Science Advisory Table. 2021;2(26). https://doi.org/10.47326/ocsat.2021.02.26.1.0.

7. Matheson FI, van Ingen T. 2016 Ontario marginalization index: user guide. Toronto, ON: St. Michael's Hospital; 2018. Joint publication with Public Health Ontario. https://www.publichealthontario.ca/-/media/documents/o/2017/on-marguserguide.pdf?la=en.

8. Lazarus JV, Wyka K, Rauh L, et al. Hesitant or Not? The Association of Age, Gender, and Education with Potential Acceptance of a COVID-19 Vaccine: A Country-level Analysis. J Health Commun 2020; 25(10): 799-807.

9. Ontario Newsroom. Ontario Easing Restrictions on Long-Term Care. https://news. ontario.ca/en/release/1000258/ontario-easing-restrictions-on-long-term-care.

10. Zhang, S. The era of mass Vaccinations is ending. May 4, 2021. The Atlantic. https://www.theatlantic.com/health/archive/2021/05/mass-vaccination-isending/618793/.

11. Government of Canada. Health Canada authorizes more flexible storage conditions for Pfizer-BioNTech COVID-19 vaccine. https://www.canada.ca/en/health-canada/ news/2021/05/health-canada-authorizes-more-flexible-storage-conditions-forpfizer-biontech-covid-19-vaccine.html.

12. Government of Canada. Vaccines for COVID-19: Shipments and deliveries. https:// www.canada.ca/en/public-health/services/diseases/2019-novel-coronavirusinfection/prevention-risks/covid-19-vaccine-treatment/vaccine-rollout.html.

13. Huynh T, Sava N, Hahn-Goldberg S, et al. Mobile on-site COVID-19 vaccination of naturally occurring retirement communities by neighbourhood risk in Toronto. Science Briefs of the Ontario COVID-19 Science Advisory Table. 2021;2(14). https://doi.org/10.47326/ocsat.2021.02.14.1.0.

14. Hunt ME, Gunter-Hunt G. Naturally Occurring Retirement Communities. Journal of Housing For the Elderly 1986; 3(3-4): 3-22.

15. Northcott, A. Shingler B. Behind the door-to-door campaign viewed as key to raising Montreal's vaccination rate. May 8,2021 . CBC. https://www.cbc.ca/news/ 
canada/montreal/montreal-covid-vaccination-rate-1.6018119. 